\title{
What Bohmian physics teaches us
}

\section{Opinion}

The last century can be called the quantum and relativity century. Because they brought us new developments in science and technology. Among the two, quantum theory is more mysterious than relativity theory, because it changed our understanding about the nature of objects in the atomic and sub-atomic domain. Furthermore, the positivistic view accompanied the standard interpretation of quantum theory. But in 1952 a casual and ontological quantum mechanics was introduced by David Bohm based on De-Broglie's ideas. The Bohm's approach has attracted attention very slowly, due to the prevalence of instrumentalism among many physicists.

The fact is that Bohmian quantum physics opens a new window towards the microscopic world from the ontological point of view. It unfolds our weakness and ignorance about the concepts of quantum world. However, up to this date, the experimental results of both approaches to quantum physics are identical. But, in the Bohmian quantum physics we can have a more imaginable picture of the world with than the standard quantum physics. I prefer to use the expression "quantum physics" rather than "quantum mechanics", because Bohmian quantum physics teaches us that we should see the universe as a whole. But the word "mechanics" is more suitable when we consider separate parts of a system. This wholeness is the subject of Bohr's complementary view too. Of course, Bohmian physics is not the last word, but it demonstrates that a causal and deterministic picture of quantum world is possible. It can be a start towards other causal quantum theories. Unfortunately, Bohmian quantum physics, like the standard quantum physics, is represented in the configuration space, as the guidance wave or wave function is represented in the configuration space. I think using a tool like wave function is the biggest obstacle towards understanding quantum world. Of course, such a tool is very imaginable, but it is hard to understand it. In the Bohmian quantum physics, however, the situation is a bit better. There, the pilot-wave has a real origin though it is represented in the configuration space. But the good thing is that the main role of the Bohm's pilot-wave is guiding particles, and statistical aspects of the pilot-wave is a secondary aspect, being due to the effects of the environment on the particle.

This means that uncertainty is not an intrinsic concept; rather, it is a mathematical tool that evades from answering the essential questions about the universe. Bohmian quantum physics describes most of the quantum phenomena by using the concept of quantum potential, without using uncertainty principle. The importance of quantum potential is that it orients our attention to the origin of quantum behavior of matter. The expression "quantum behavior of matter" has no room in the standard quantum physics, as we replace all physical quantities by their operational equivalents and make an algorithm to relate some initial data to some final data, accompanied by an intrinsic uncertainty. But in Bohmian physics, we have a causal explanation for quantum tunneling, the trajectories of particles in the double slit experiment, etc.

It is sometimes not mentioned that Bohmian quantum potential is an essential concept. But, in our view, this is not a correct view. We could get Bohmian quantum potential through a different starting point, without using Schrodinger's equation or postulating a wave-

\author{
Volume I Issue 3 - 2017
}

\section{Faramarz Rahmani}

School of Physics, Institute for Research in Fundamental Science (IPM), Iran

Correspondence: Faramarz Rahmani, School of Physics, Institute for Research in Fundamental Science (IPM), Tehran, Iran, Email faramarzrahmani@ipm.ir

Received:September 23, 2017 | Published: October 03, 2017

function from the beginning. For example, we have demonstrated that if we consider Mach's principle, then by using some postulates we can get Bohmian-type terms in the Hamilton-Jacobi equation of a relativistic spinless particle. This is a simple work and could be continued further.

If we replace the wave function with a physical property or physical force, what happens to some aspects like non-locality? How a property of quantum physics like non-locality can be included in a real universe? In other words, how can it be interpreted in a real fourdimensional space? Bohmian physics, teaches us that a local view of universe is not sufficient, as we have learned from the Bell's work. How, the whole of a system affects one of its parts? When we say "how" we are asking for the procedure from the ontological view, and not merely using a mathematical tool like a non-local pilot-wave.

Bohmian quantum physics has not been developed yet in the context of field theory coherently. For scalar fields it has a functional formulation but this is not the case for fermion fields. In the Bohmian quantum mechanics, a wave function, with a real origin, guides a real entity, a particle. But in the case of quantum fields what is the real field for fermions? We are familiar with the real field of photon e. $g$ electromagnetic field which has classical analogues and is detectable. But for fermions (e.g. electrons) we have no such real detectable field. In standard quantum mechanics, there is no such problem, because all things are adapted with an operational formalism in an abstract Hilbert space. But, when we want to understand universe ontologically, these problems arise. May be fermions are only particles and bosons are fields, as once Dirac suggested. Then, how the creation or annihilation of a fermion is described, when its four-velocity always is time-like?

Such questions come up when we want to understand nature causally. Naturally, answering these questions is not easy, but the interesting aspect of physics is the difficulty in understanding our universe exactly.

\section{Acknowledgments}

None.

\section{Conflicts of interest}

The author declares there is no conflict of interest. 